\title{
Vivienda Social y teorías de la vida cotidiana: aproximaciones al caso Barrio Parque Los Andes
}

\section{Social Housing and theories of daily life: approaches to the Barrio Parque Los Andes case}

Fabri, María Eugenia

Arquitecta, Laboratorio de tecnología y gestión habitacional, Facultad de arquitectura y urbanismo, Universidad Nacional de La Plata, Buenos Aires, Argentina.

eugenia.fabri@gmail.com

\section{RESUMEN}

Con el objeto de identificar parámetros que aporten a una valoración transdisciplinar de la Vivienda Social, este trabajo recupera las nociones centrales de las teorías de la vida cotidiana. En términos metodológicos, se considera como caso de estudio el Barrio Parque Los Andes, conjunto habitacional construido en los años 20 , singular por su potencial cualificador del espacio urbano. Como uno de los referentes paradigmáticos, que forman parte del cuerpo teórico de la disciplina, aporta miradas alternativas a la producción habitacional contemporánea aun cuando su planteo original remite a otros modos de habitar. Se hipotetiza que la eficiencia del caso obedece al adecuado entramado que propone su red de relaciones sobre el espacio urbano, tal como verifica la construcción cartográfica que se expone en el desarrollo.

Se pretende entonces interpelar la propuesta desde los vigentes postulados teóricos, ponderando su concepción integral en relación con la interfase vivienda-ciudad.

\section{ABSTRACT}

In order to identify parameters that contribute to a transdisciplinary assessment of Social Housing, this work recovers the central notions of the theories of everyday life.

In methodological terms, the Barrio Parque Los Andes is considered as a case study, a housing complex built in the 1920s, unique for its potential qualifying urban space. As one of the paradigmatic references, which are part of the theoretical body of the discipline, it provides alternative views to contemporary housing production even when its original approach refers to other ways of living. It is hypothesized that the efficiency of the case obeys the adequate framework proposed by its network of relationships on the urban space, as verified by the cartographic construction that is exposed in the development.

The intention is then to question the proposal from the current theoretical postulates, weighing its integral conception in relation to the housing-city interface.

PALABRAS CLAVE: vivienda pública - proyecto - género

KEYWORDS: public housing - planning - gender

FECHA DE RECEPCIÓN: 21/9/2021 | FECHA DE ACEPTACIÓN: 9/11/2021

DOI: $\underline{\text { http://dx.doi.org/10.30972/arq.0185676 }}$ 
Vivienda Social y teorías de la vida cotídiana: aproximaciones al caso Barrio Parque Los Andes

\section{INTRODUCCIÓN}

En la ciudad de Buenos Aires, más específicamente en el barrio Chacarita, a pocas cuadras del cementerio, frente al Parque Los Andes, encontramos la Primera Casa Colectiva Municipal (Figura 01), proyectada por el Arq. Fermín H. Bereterbide (1899, Rosario-1979, Buenos Aires) en el año 1924 para un concurso estatal que buscaba extender la ciudad hacia sitios remotos para completar la trama urbana y descomprimir el hacinamiento de los conventillos del centro porteño. Concurso resuelto por la Municipalidad de Buenos Aires, con parte de los fondos provenientes del empréstito para fomento urbano, emitido por Ordenanza del 17 de agosto de 1923. Esta obra se levantó sobre un terreno municipal de 13.888 metros cuadrados, limitado por las calles Leiva, Rodney, Guzmán y Concepción Arenal, en un término de 16 meses.
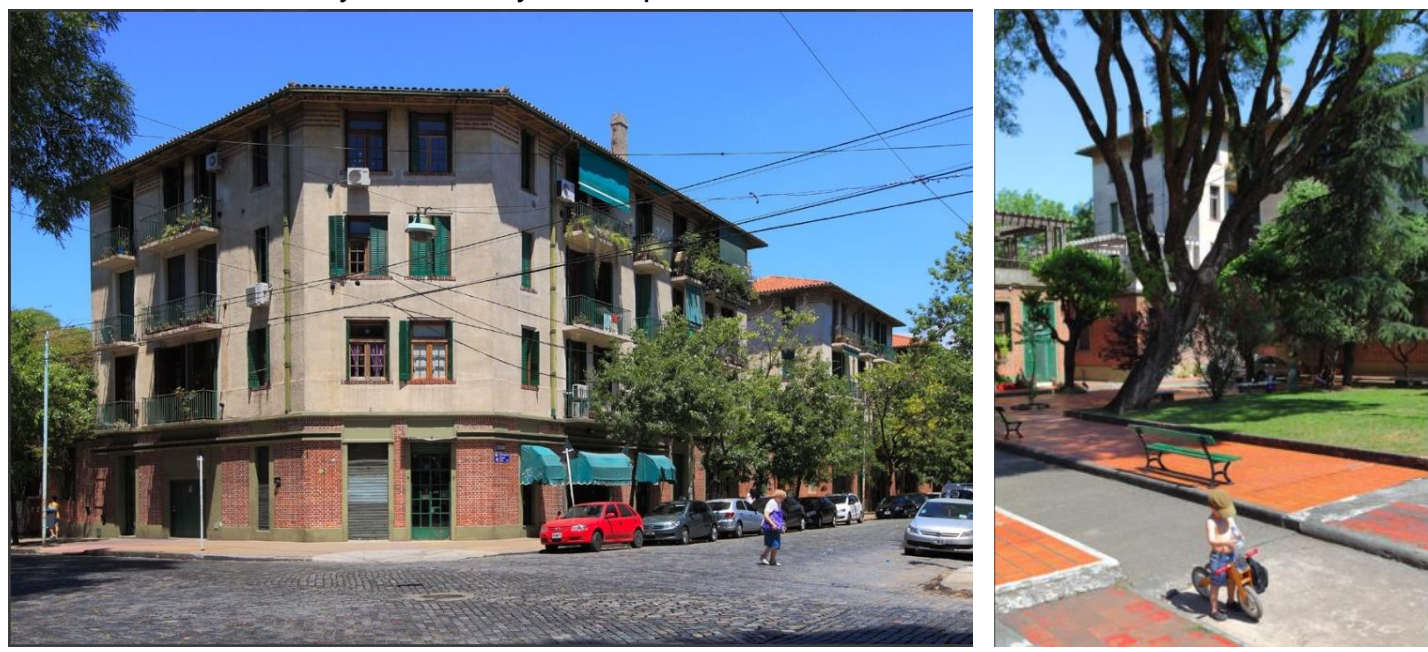

Figura 01: Fotografía del conjunto. Figura 02: Fotografía de jardín interior.

Fuente: Corral, Pablo; Revista SCA

La ideología socialista de Bereterbide impregna su obra con criterios de justicia e igualdad social, a lo que se suman ideas higienistas de vanguardia en Europa: higiene física (asoleamiento, ventilación e iluminación naturales) e higiene moral (todas las unidades tienen el mismo criterio funcional, de confort y de relación con los espacios verdes).

A lo largo de su carrera, fueron tema de estudio, la vivienda colectiva, vivienda popular y vivienda mínima, forma de ocupación de los lotes, tejidos urbanos, loteos y amanzanamiento. "Sus reflexiones sobre vivienda popular fueron volcadas en diversos artículos publicados en Nuestra Arquitectura, Revista de Arquitectura y Revista del CACYA y, finalmente, en su libro "La vivienda popular" (1959), donde abordaba tanto la arquitectura habitacional como sus formas de gestión y financiamiento". (LIERNUR, 2004)

“... La gente siempre querrá saber, lo necesita, dónde está la entrada de su casa, su vereda, su grupo vecino inmediato, querrá confirmarlo en la costumbre cotidiana, y será capaz de aceptar versiones nuevas siempre que éstas sean capaces de cumplir, aunque sea de otra manera, dichas funciones tradicionales. A este manejo de lo socialmente instaurado en la memoria colectiva y los modos de vida no fueron capaces de responder aquellas macro arquitecturas de los 60 y los 70". Fermín Bereterbide.

"El conjunto Los Andes, que tiene como antecedente un complejo similar en el barrio de Flores, que el mismo autor ganó por concurso en 1920, se integró morfológicamente a la ciudad con una cuota de utopía social. Sus costos de ejecución fueron muy altos, por tener un importante equipamiento comunitario y un $63 \%$ de superficie destinada a parque y expansión (Figura 02). 
Por esta razón, otros concursos ganados por el autor no se construyeron. Se trató de adaptaciones de la misma idea a las particularidades de terrenos que se emplazaban en Palermo y Flores". (GONZÁLEZ MONTANER, 2005)

\section{METODOLOGÍA}

Este trabajo parte de un estudio de caso, significativo en el marco de la producción habitacional estatal del siglo XX, para cuyo análisis se recurre a las siguientes técnicas metodológicas:

1. Discusión bibliográfica a partir de autores referentes en materia de teorías de la vida cotidiana (entre otros Ciocoletto, Bereterbide, Jacobs) que aportaran precisiones en torno a diferentes escalas obtenidas de la dinámica temporal de tránsito peatonal, a saber, escala de vecindario, escala barrial y escala suprabarrial. A su vez, los aportes del Col lectiu Punt $6^{1}$ diferencian tres variables de análisis a tener en cuenta: equipamiento cotidiano, espacios de relación y transporte. 2. Construcción de cartografías temáticas a partir de las variables y escalas mencionadas anteriormente.

\section{DESARROLLO}

"Antes de comenzar a analizar el Conjunto Parque Los Andes (Figura 03), es importante conocer que el programa del concurso fijaba gran parte de las condiciones del conjunto; planteaba que la manzana debía tomarse completa, que la superficie del terreno libre debía ser igual o superior al $50 \%$ de la superficie del predio (Figura 04) y que todos los proyectos debían resultar en conjuntos con 1000 hab./Ha de densidad. También se aconsejaba suprimir los "pozos de aire y luz", "los corredores cerrados" y utilizar escaleras que sirvieran a un número reducido de unidades." (PRESSLER, 2010)

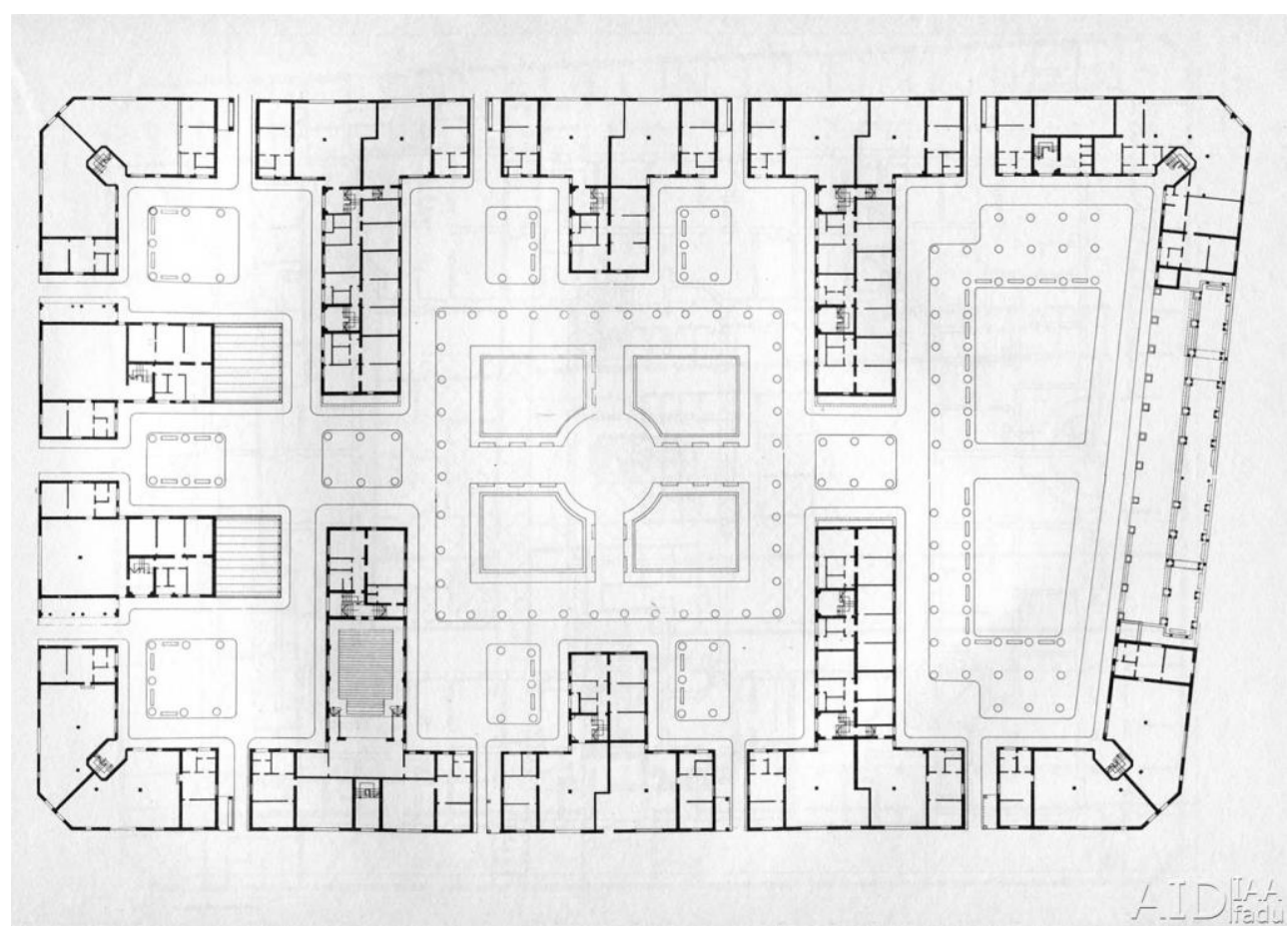

1 El Col lectiu Punt 6 es una cooperativa de trabajo asociado constituida por arquitectas, sociólogas y urbanistas de procedencias diversas, formada en el año 2005. Trabajan por repensar los espacios domésticos, comunitarios y públicos desde una perspectiva feminista, con más de 400 proyectos realizados en el ámbito local, estatal e internacional. 
Vivienda Social y teorías de la vida cotídiana: aproximaciones al caso Barrio Parque Los Andes

Figura 03: Plano de conjunto. Dibujo de línea negra sobre fondo blanco.

Fuente: AID - FADU - Taller Tony Díaz. "Relevamientos. 1984-1985" FAU - UNBA. Buenos Aires, 1985.

Si nos adentramos en las actuales teorías de la vida cotidiana que intentan transformar las áreas residenciales monofuncionales para mejorar la calidad de vida de las personas, vamos a observar que este Barrio, inaugurado el día 6 de octubre de 1928, y administrado por los vecinos desde 1972 cumple con todas las características indispensables para que los residentes puedan vivir en una comunidad que satisfaga sus necesidades tanto propias como políticas, productivas y reproductivas. Las cuatro esferas que el Col lectiu Punt 6 considera imprescindibles para el desarrollo de la vida cotidiana.

Entre los actuales residentes que habitan esta casa colectiva, encontramos artistas, arquitectos y arquitectas, escritores, que tienen sus talleres y estudios en sus viviendas. Docentes que dan clases a las infancias vecinas, enseñando arte, música y dando apoyo escolar, cumpliendo así parte de su día laboral en el mismo barrio que habitan. Los parques y jardines son testigos del desarrollo tanto de la esfera propia como la reproductiva ya que se realizan en ellos reuniones de vecinos-amigos, mamás y papás con sus bebés, sus hijos e hijas pequeños y hasta se celebran fiestas y fechas importantes, como fin de año, donde entre todos despliegan mesas y hacen grandes asados compartidos.

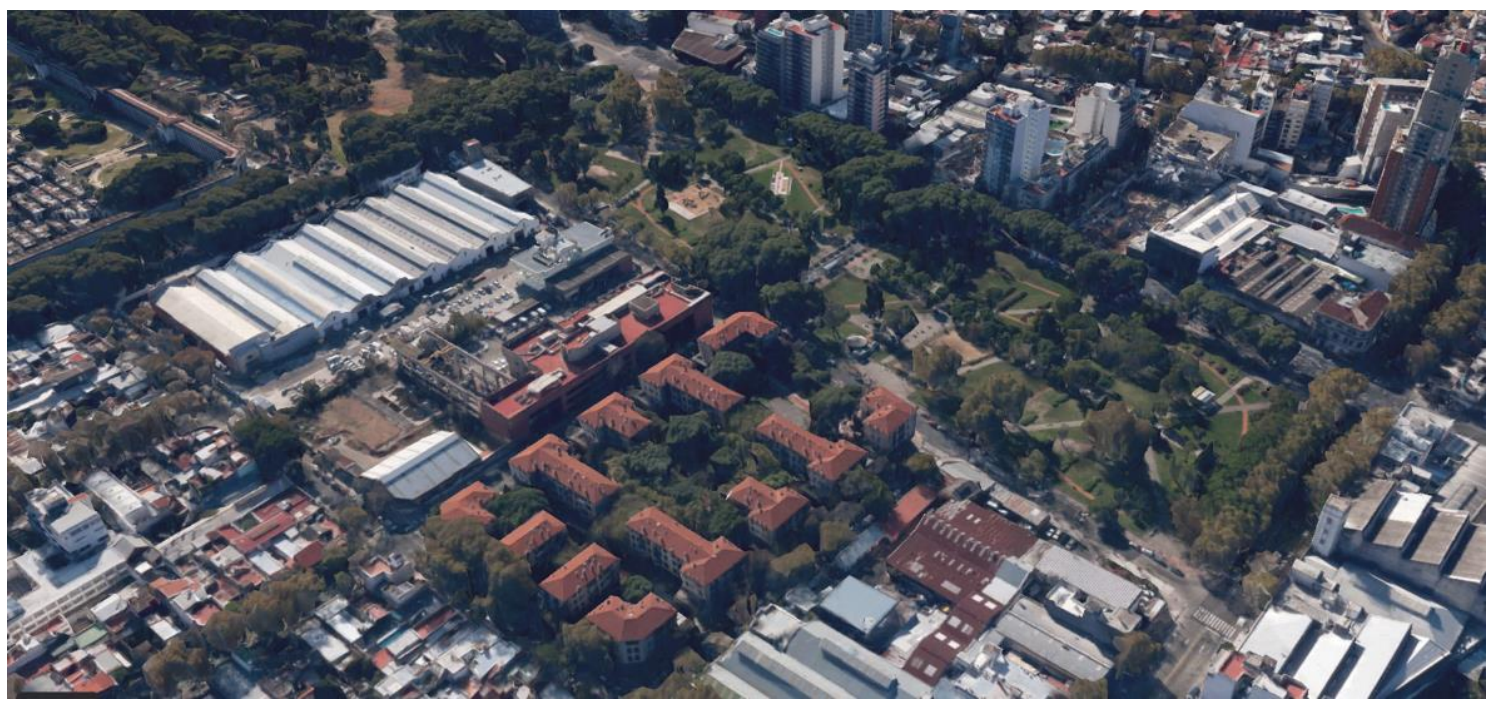

Figura 04: Jardines interiores y apertura del conjunto hacia el Parque los Andes.

Fuente: Imagen satelital extraída de Google Earth.

También la esfera política es indispensable para que el barrio no pierda su esencia, está organizado en comisiones con un presidente, secretario y tesorero que trabajan ad honorem posibilitando que la comunidad funcione, que se mantenga el conjunto, tanto la construcción como su espíritu.

Según la discriminación realizada por Adriana Ciocoletto en su tesis, el Barrio y la red cotidiana (Figura 05) se divide en tres escalas, vecindario, barrio y suprabarrial. Por lo mencionado anteriormente podemos afirmar que la escala de vecindario, que es el "espacio que se encuentra en la inmediatez de la vivienda, que se comparte con las personas vecinas y donde es posible encontrarse y socializar" (CIOCOLETTO, 2014), está completa. La escala de barrio es el "espacio ampliado del vecindario donde se realizan las tareas cotidianas. Es donde, en una situación óptima, se encuentran los espacios de relación, equipamientos cotidianos, comercios necesarios para el día a día y transporte público. Puede medirse aproximadamente en un radio de $10 \mathrm{~min}$ a pie, que correspondería al trayecto que una persona sin dificultades puede realizar" (CIOCOLETTO, 2014). En este caso podemos notar que si algún elemento faltase en el conjunto 
para cumplimentar las actividades cotidianas, a pesar de ser un lugar tranquilo y aparentemente alejado de la vorágine del microcentro porteño, el mismo se encuentra inmerso en el centro de la ciudad a escasos metros de Corrientes y Belgrano y rodeado de avenidas donde encontrar el equipamiento necesario para cualquier actividad ya sea administrativa, de servicio, salud, educación, entretenimiento, transporte público entre otras.

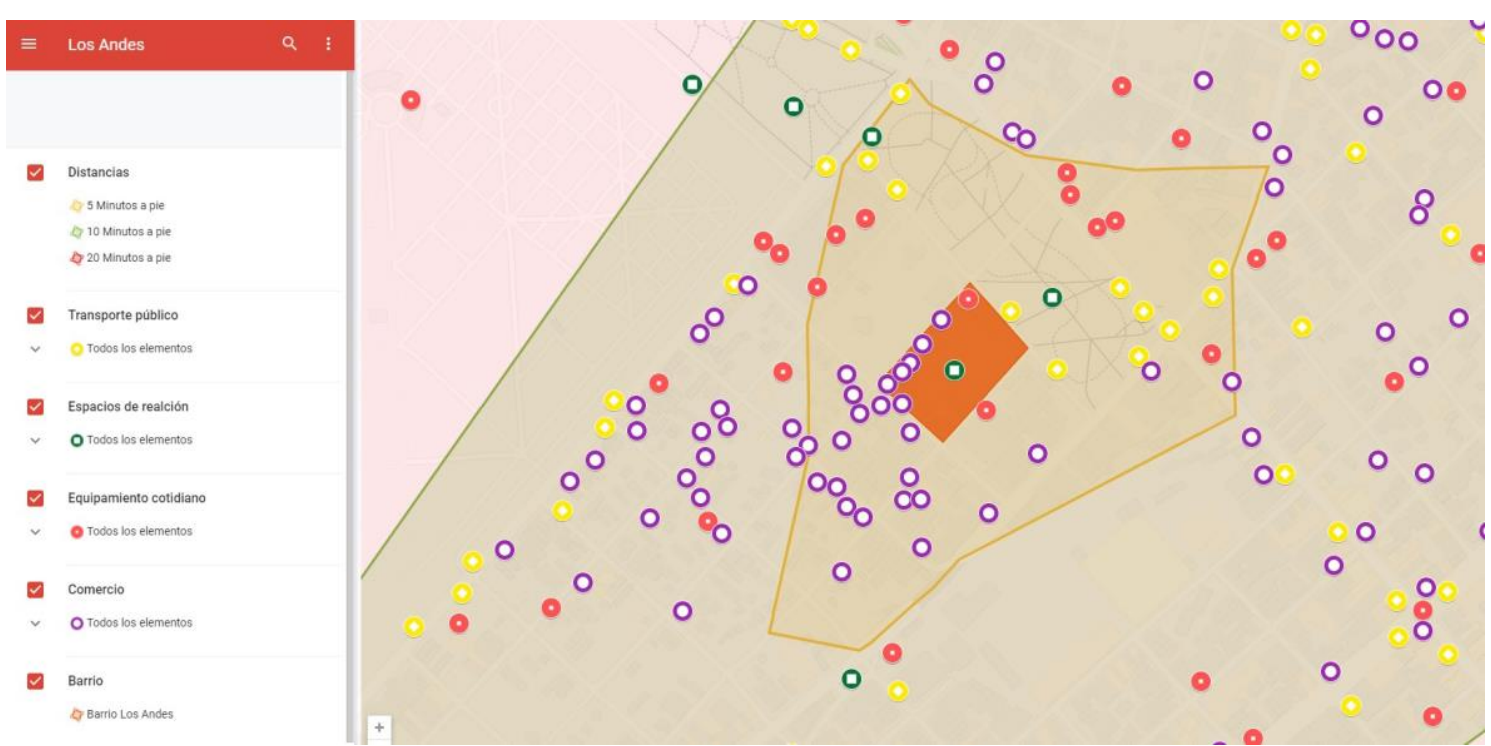

Figura 05: Barrio y red cotidiana. Transporte público, espacios de relación, equipamiento cotidiano y comercios en las cercanías del barrio, en un radio de dos distancias diferentes recorridas a pie, 5 minutos y 10 minutos.

Fuente: Elaboración propia con datos extraídos de Google Maps.

La tercera escala, suprabarrial (Figura 06), por lo visto anteriormente está más que completa, y se define como el "espacio que se encuentra fuera de la escala de barrio y donde se hallan el resto de espacios de relación, equipamientos y comercios que no necesitan estar en la escala de barrio por no estar relacionados con dependencias familiares o de la unidad de convivencia. Puede medirse aproximadamente en un radio de $20 \mathrm{~min}$ a pie, considerando que es el trayecto que una persona sin dificultades puede recorrer y/o cubrir en transporte público" (CIOCOLETTO, 2014).
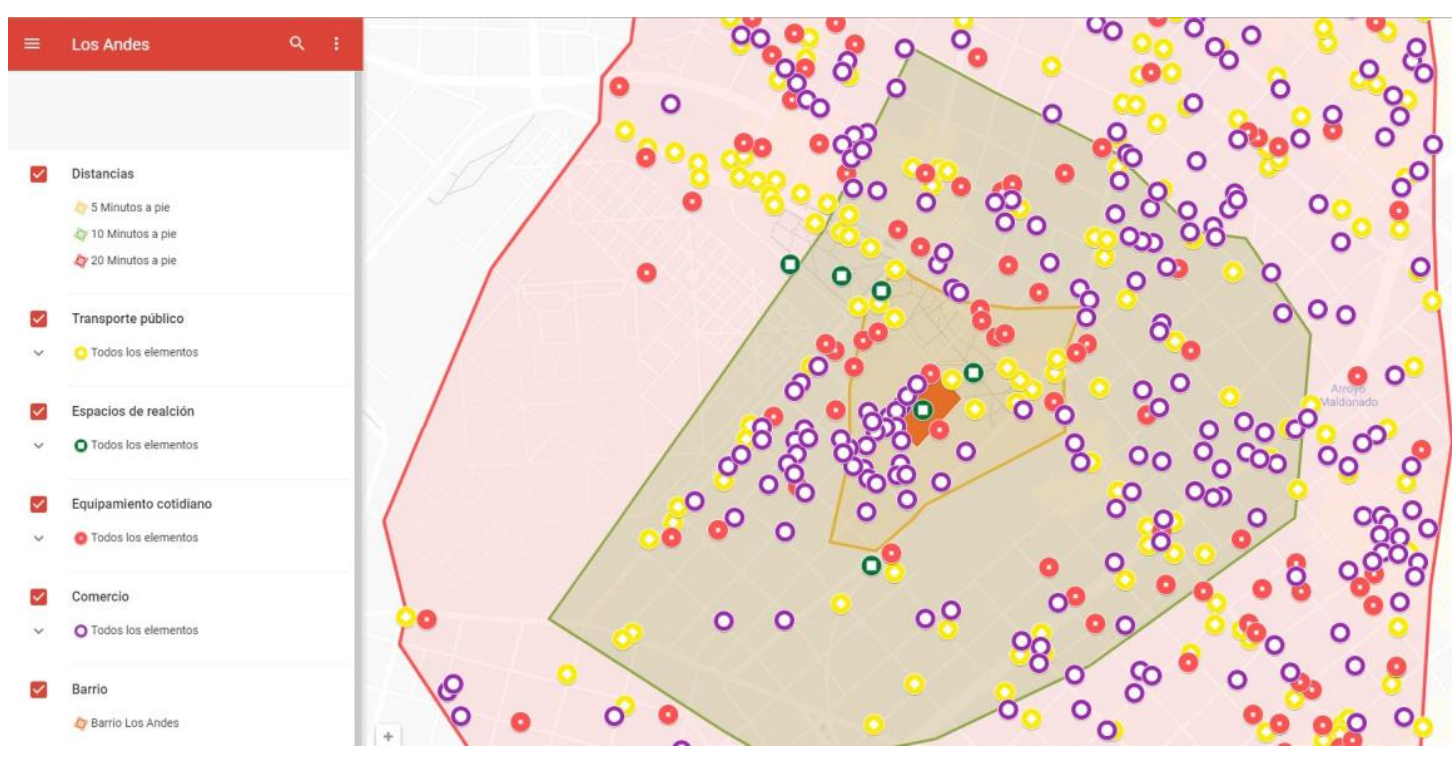
Vivienda Social y teorías de la vida cotídiana: aproximaciones al caso Barrio Parque Los Andes

Figura 06: Barrio y red cotidiana. Transporte público, espacios de relación, equipamiento cotidiano y comercios en las cercanías del barrio, en un radio de tres distancias diferentes recorridas a pie, 5 minutos, 10 minutos y 20 minutos. Fuente: Elaboración propia con datos extraídos de Google Maps.

Los Equipamientos cotidianos (Figura 07) son "aquellos equipamientos que se utilizan diariamente y que son indispensables como soporte para el desarrollo de la vida cotidiana en todas las etapas vitales y para la mejora de la calidad de vida de las personas. Son también los espacios de referencia de una comunidad donde se genera la convivencia, el intercambio, la socialización y la ayuda mutua. Se considera que hay un mínimo de equipamientos que deben estar dentro de la red cotidiana, pensados para responder a las necesidades de las personas, a partir de programas, pero que no necesariamente tienen que localizarse en un edificio específico, es decir, que pueden existir espacios polifuncionales que respondan a más de una de estas necesidades. También puede ampliarse el listado de mínimos según la particularidad de cada barrio o municipio y la forma de resolverlo dependerá en cada caso del tamaño y cantidad de población" (CIOCOLETTO, 2014)

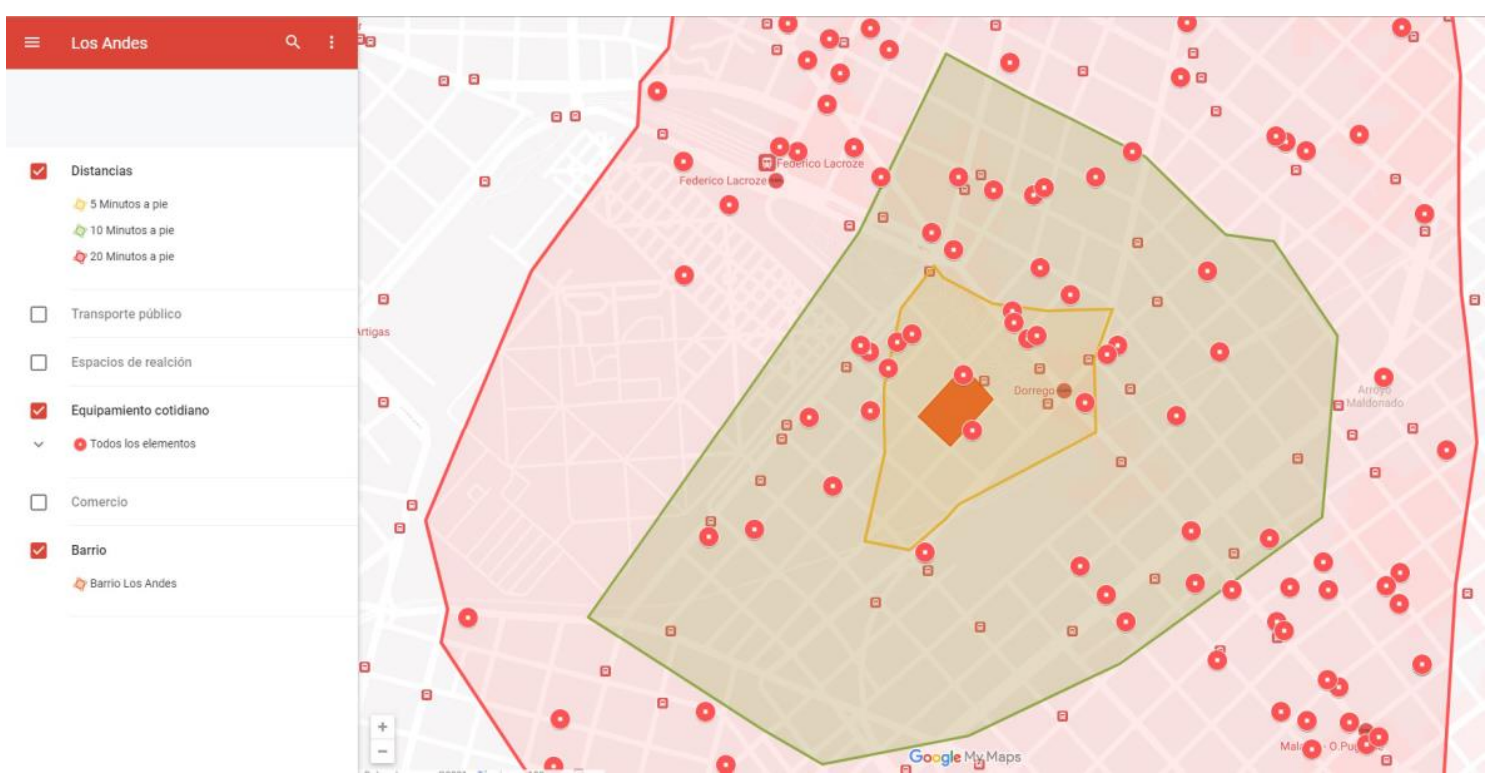

Figura 07: Equipamientos cotidianos en un radio de tres distancias diferentes recorridas a pie, 5 minutos, 10 minutos y 20 minutos. Fuente Elaboración propia con datos extraídos de Google Maps.

El complejo consta (originalmente) de 130 departamentos de 3,4 y 5 ambientes distribuidos en 12 pabellones de planta baja y 3 pisos, 23 locales comerciales, hoy muchos de ellos devenidos en viviendas, baños públicos, lavaderos, salón de espectáculos (Figura 08), biblioteca pública, jardín de infantes y más de $7.000 \mathrm{~m} 2$ de espacios verdes comunes entre parques, jardines, juegos para las infancias y espacios deportivos y terrazas privadas (Figura 09). Además, el edificio cuenta con ciertos adelantos tecnológicos para la época como agua caliente, conductos para la eliminación de residuos, teléfono, y ventilaciones para estufas a carbón (Figura 10).

Las unidades están ubicadas a lo ancho de los edificios, lo que permite que las habitaciones se distribuyan de un lado y los servicios hacia el otro, recurso que, a su vez, facilita la ventilación y la independencia de las unidades, separándolas mediante escaleras o dobles muros de ladrillo hueco. Están compuestas por un estar comedor, con expansión; 2, 3, 4 o 5 habitaciones: una cocina equipada con un artefacto a gas, un baño con bañera, lavatorio e inodoro, un lavadero y un cuarto de guardado. Fueron diseñadas para brindar vivienda a familias con un promedio de 4 hijos cada una (brindando así unidades para familias de 2 a 6 hijos). Es decir, se calculaba la cantidad de habitantes de la casa colectiva entre 180 y 200 adultos y entre 360 y 400 niños. Las 
Vivienda Social y teorías de la vida cotidiana: aproximaciones al caso Barrio Parque Los Andes

Fabri

unidades son amplias y con detalles de confort poco comunes para la época en viviendas económicas.
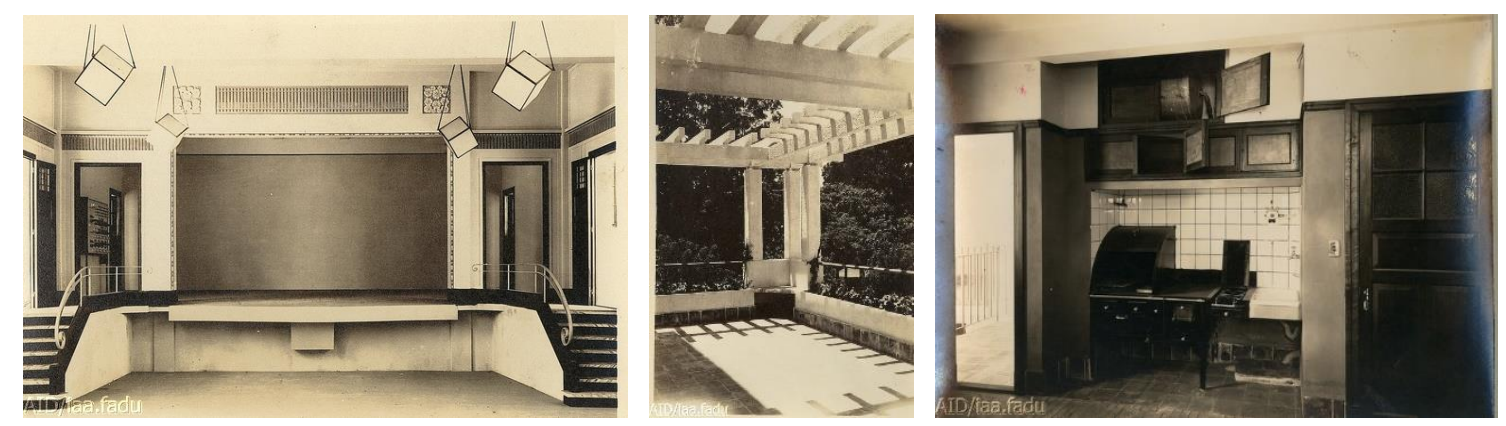

Figura 08: Escenario. Figura 09: Terraza y pérgola. Figura 10: Cocina de vivienda. Fuente: AID - FADU - UBA Colección personal del Arq. Juan Molina y Vedia

Todos los departamentos cuentan con ventilación e iluminación natural, los bloques están distribuidos de tal manera que no arrojan sombras entre sí ni bloquean las visuales hacia la calle o hacia el parque central (Figura 11) consiguiendo de esta manera que las personas puedan ver desde sus ventanas o balcones los espectáculos organizados en la fuente central, los partidos de futbol en la cancha, a sus hijos jugando en los jardines.

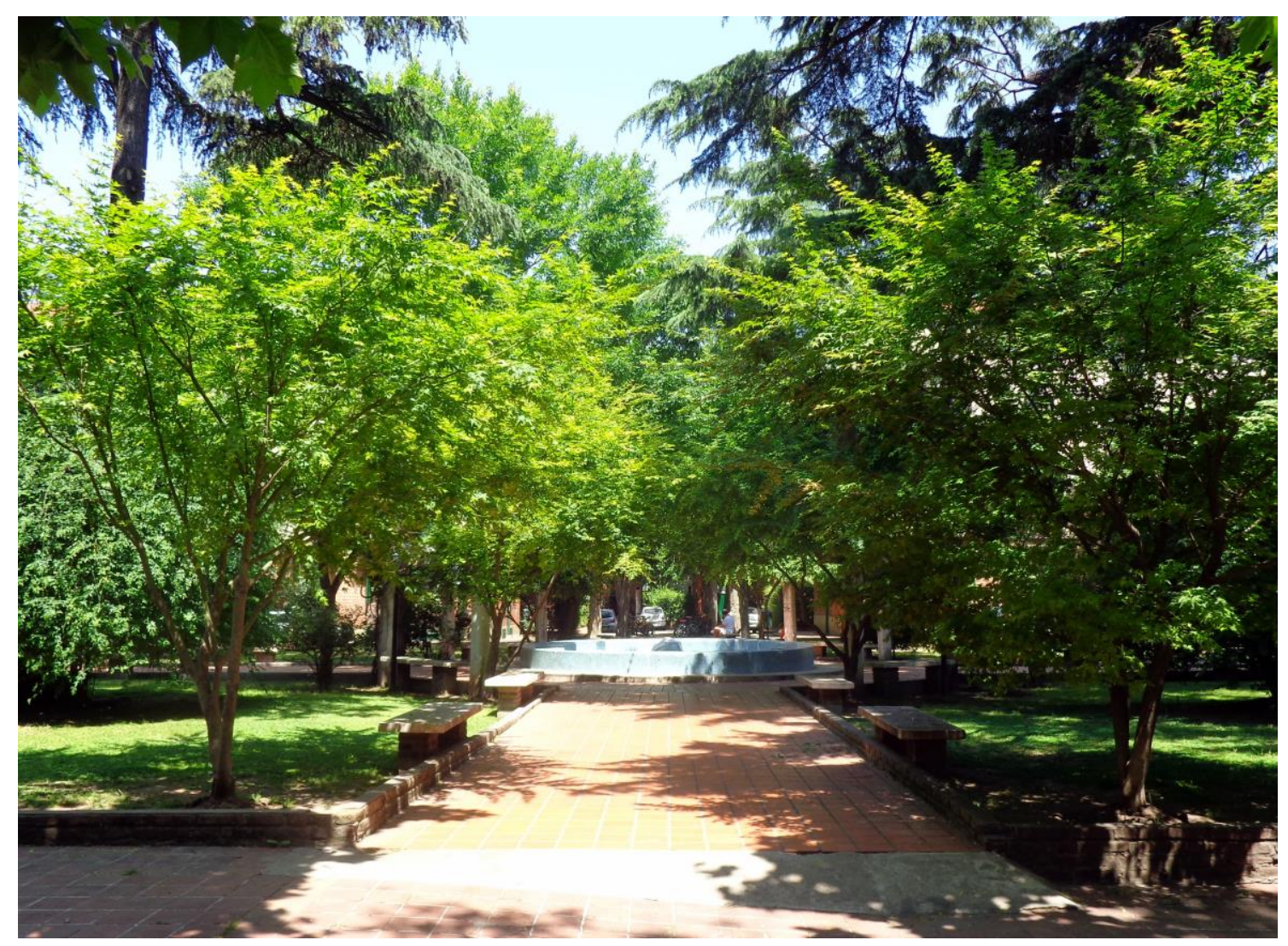

Figura 11: Fuente y jardín central.

Fuente: Roberto Fiadone - Wikipedia. 
Vivienda Social y teorías de la vida cotídiana: aproximaciones al caso Barrio Parque Los Andes

Esto nos recuerda otra gran urbanista, Jane Jacobs y su teoría "Ojos en la calle": "Tiene que haber ojos en las calles, ojos pertenecientes a los que podríamos llamar los propietarios naturales de la calle. Los edificios deben estar orientados hacia la calle para garantizar la seguridad de los residentes y extranjeros. No pueden dar la espalda o tener lados en blanco y dejarlos ciegos". (JACOBS, 1961) En esta obra, Jacobs explica que uno de los objetivos más importantes de los espacios públicos es que las personas se sientan cómodas, seguras y protegidas, aunque no se conozcan entre sí. Esta situación se lograría si se cumple la teoría de "ojos en la calle". La autora y activista, defendía los usos mixtos, el espacio público y la diversidad dentro de las ciudades, todos elementos que hallamos sin mayor esfuerzo en la arquitectura de vivienda social de Beretervide.

Los espacios de relación (Figura 12) "Son aquellos espacios donde las personas pueden interactuar en el momento de realizar actividades cotidianas en su entorno urbano (barrio), principalmente las que tienen que ver con el cuidado del hogar y de otras personas, que permiten el fortalecimiento de las redes sociales y de ayuda mutua. Son también los espacios de ocio, juego y encuentro que posibilitan que las personas de una comunidad convivan, se conozcan y aprendan de la diversidad que cada una aporta a la sociedad". (CIOCOLETTO, 2014) En este caso el conjunto cuenta con varios metros cuadrados destinados a las relaciones entre vecinos, la fuente es el centro de la casa común y es punto de encuentro, rememora los patios de los conventillos de la época donde toda la vida se desarrollaba en el patio central, de manera comunitaria, pero con la diferencia de la calidad espacial diseñada por Bereterbide, la ventilación, el asoleamiento, los jardines colmados de vegetación, rodeados por los departamentos de ambientes generosos y bien iluminados.

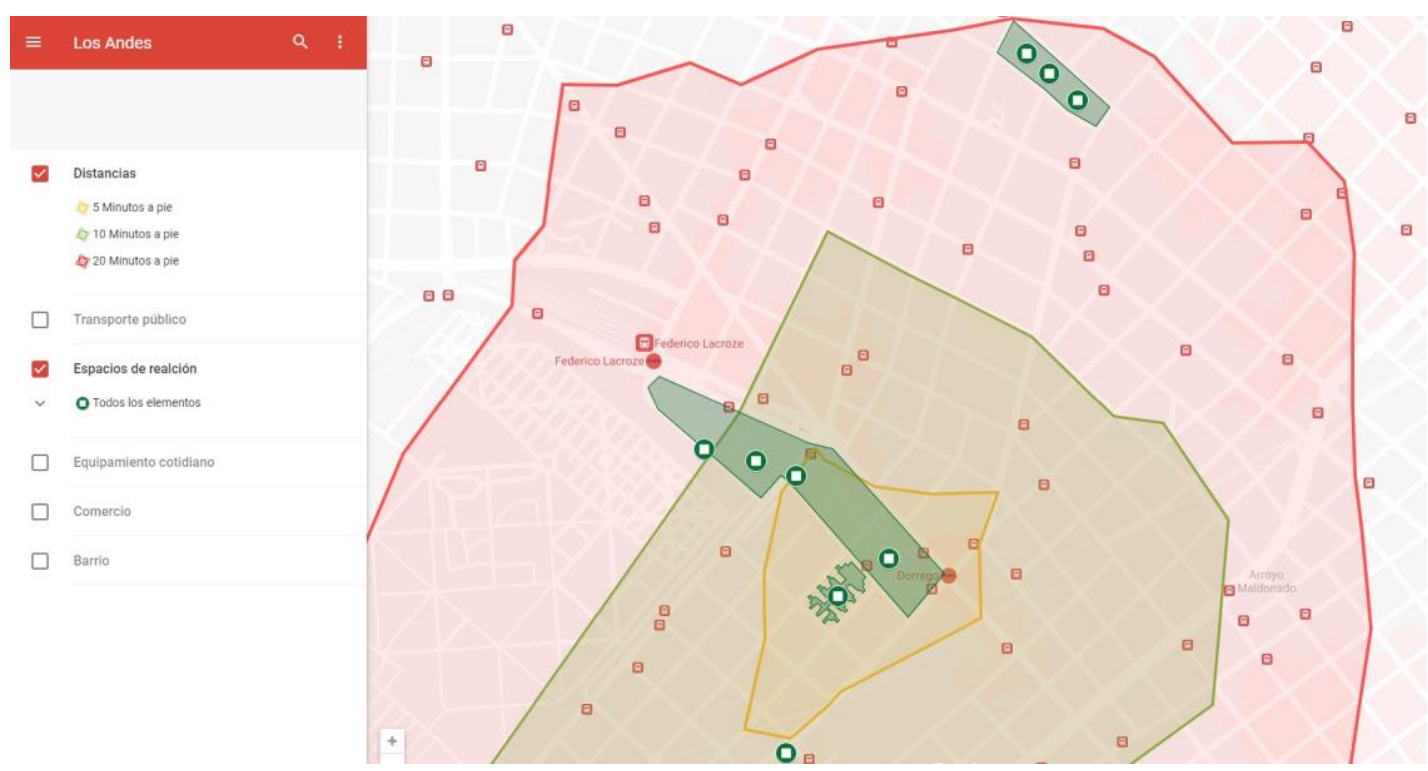

Figura 12: Espacios de relación en un radio de tres distancias diferentes recorridas a pie, 5 minutos, 10 minutos y 20 minutos.

Fuente Elaboración propia con datos extraídos de Google Maps.

\section{CONCLUSIONES}

El análisis del caso corrobora entonces, que los servicios habitacionales que ofrece la Vivienda Social, en relación con sus valores de proximidad, dependen de su posición en una configuración espacial urbana determinada (YUJNOVSKY, 1984). En este sentido, con las acciones orientadas a la construcción de viviendas, el Estado incide directamente en la dinámica urbana. Sin embargo, esto no garantiza que, en estos modelos de urbanización social, las posibilidades 
resulten equitativas para todos los habitantes. Es decir, todos estos análisis cambian dependiendo del usuario, de su género, su edad, su movilidad, etc. No es lo mismo recorrer estos trayectos solo, o acompañado, siendo adulto o niño, o adulto mayor, siendo mujer u hombre, de día o noche. Es importante tener en cuenta a todas las personas a la hora de realizar un análisis de la interfase vivienda-ciudad. Ya que subir con un cochecito al subte, cruzar una vía teniendo movilidad reducida, o caminar desde la estación al hogar cuando el día está terminando puede dilatar los minutos o acortarlos de manera sustancial. “...La Poeta Adrienne Rich (...) habla de una política de plantear preguntas de mujeres $(\mathrm{RICH}, 2001)$. No se trata de preguntas esencialistas o basadas en la errónea pretensión de una definición biológica de la femineidad. Se trata, por el contrario, de preguntas que emergen de la experiencia cotidiana y corporal de quienes se incluyen a sí mismas en la cambiante y dinámica categoría de mujeres. Para nosotras, la vida de ciudad plantea preguntas que ya llevan demasiado tiempo sin respuestas." (KERN, 2019)

\section{BIBLIOGRAFÍA}

Ciocoletto, Adriana y Col-lectiu Punt 6 (2014) Espacios para la vida cotidiana. Auditoría de Calidad Urbana con perspectiva de Género. Barcelona: Editorial Comanegra.

González Montaner, H. A. (2005) Vanguardias Argentinas. Obras y movimientos en el siglo XX. Vol. 02. Arquitectura 1930/1950 Buenos Aires: AGEA.

Jacobs, Jane (1961) Muerte y vida en las grandes ciudades. The Death and Life of Great American Cities. Ed. 2011 España: Gráficas Lizarra.

Kern, Leslie (2019) Ciudad Feminista. La lucha por el espacio en un mundo diseñado por hombres. Feminist city. A field guide. Canadá Between the Lines. Tr. CABA (2020): Ediciones Godot.

Liernur, Jorge F. y Aliata, Fernando (2004) Diccionario de Arquitectura en la Argentina. Buenos Aires: AGEA

Pressler, M. Victoria (2010) Revista PLOT N01. Buenos Aires.

Rich, Adrienne, (2001) "Apuntes para una política de la posición”, en: Sangre, pan y poesía. Prosa escogida: 1979-1985. Barcelona: Icaria.

Yujnosvky, Oscar (1984) "Claves políticas del problema habitacional argentino 1955-1981." Buenos Aires: Grupo Editor Latinoamericano. 
